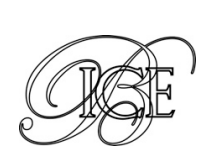

\title{
LAS ASAMBLEAS DE PRIMAVERA DEL FONDO MONETARIO INTERNACIONAL DE ABRIL DE 2015
}

Las Asambleas de Primavera de 2015 se celebraron en un contexto de mejora económica en la zona euro y Japón, pero con una clara desaceleración en los países emergentes. La situación monetaria y financiera es excepcional, con muchos tipos de interés en niveles mínimos históricos y niveles de inflación muy bajos tras la fuerte caída de los precios del petróleo y las materias primas. La reforma del gobierno del FMI, acordada en 2010, continúa bloqueada, pendiente de la ratificación de los Estados Unidos. En estas Asambleas se ha acordado otorgar más tiempo a los EEUU, puesto que no hay acuerdo sobre posibles alternativas.

Palabras clave: instituciones financieras multilaterales, política económica, tipos de interés. Clasificación JEL: E52, F33.

\section{Introducción}

Las Asambleas del Fondo Monetario Internacional (FMI), celebradas conjuntamente con el Banco Mundial, reúnen cada seis meses a los ministros de Economía y Hacienda o Finanzas de los 188 países miembros. Constituyen una oportunidad para discutir a nivel ministerial los principales desarrollos económicos y el signo de las políticas económicas, así como para dar orientaciones políticas al trabajo de las instituciones financieras multilaterales.

Una de las principales novedades de las últimas Asambleas de abril de 2015 es que el gobernador del Banco de México, D. Agustín Carstens, ha presidido las reuniones del Comité Monetario y Financiero Internacional (CMFI o IMFC por sus siglas en inglés), que reúne a 24 de los principales

\footnotetext{
* Secretaría General del Tesoro y Política Financiera. Este artículo ha sido elaborado por Miguel Ángel Martínez Rolland.

Versión de junio de 2015.
}

ministros y gobernadores de bancos centrales para fijar las orientaciones del trabajo del FMI. Es la primera vez que un ministro o gobernador de Iberoamérica ${ }^{1}$ preside este comité, y Carstens ha sido elegido por ser un gobernador de reconocido prestigio, que conoce bien la institución por haber sido uno de los subdirectores gerentes del Fondo.

\section{Situación económica internacional}

En anteriores Asambleas, un tema recurrente ha sido la debilidad del crecimiento en la zona euro y Japón, que contrastaba con un crecimiento cada vez más fuerte en Estados Unidos, y un impresionante crecimiento en los países emergentes. Ahora bien, la zona euro retorna, según las previsiones del FMI, a crecimientos superiores al 1 por 100, todavía modestos, pero en clara recuperación. España $\triangleright$

\footnotetext{
1 Cabe señalar que el español Carlos Solchaga presidió entre 1991 y 1993 el antecesor del CMFI, el llamado «Comité Interino».
} 


\begin{tabular}{|c|c|c|c|c|}
\hline \multicolumn{5}{|c|}{$\begin{array}{c}\text { CUADRO } 1 \\
\text { CRECIMIENTO E INFLACIÓN }\end{array}$} \\
\hline \multicolumn{5}{|c|}{ Crecimiento del PIB (\% variación interanual) } \\
\hline & 2013 & 2014 & 2015(P) & 2016 (P) \\
\hline  & $\begin{array}{r}1,4 \\
2,2 \\
-0,5 \\
0,2 \\
0,3 \\
-1,7 \\
-1,2 \\
1,6 \\
1,7\end{array}$ & $\begin{array}{r}1,8 \\
2,4 \\
0,9 \\
1,6 \\
0,4 \\
-0,4 \\
1,4 \\
-0,1 \\
2,6\end{array}$ & $\begin{array}{l}2,4 \\
3,1 \\
1,5 \\
1,6 \\
1,2 \\
0,5 \\
2,5 \\
1,0 \\
2,7\end{array}$ & $\begin{array}{l}2,4 \\
3,1 \\
1,6 \\
1,7 \\
1,5 \\
1,1 \\
2,0 \\
1,2 \\
2,3\end{array}$ \\
\hline $\begin{array}{l}\text { Mercados emergentes y economías en desarrollo } \\
\text { Rusia } \\
\text { Asia } \\
\text { China } \\
\text { India }\end{array}$ & $\begin{array}{l}5,0 \\
1,3 \\
7,0 \\
7,8 \\
6,9\end{array}$ & $\begin{array}{l}4,6 \\
0,6 \\
6,8 \\
7,4 \\
7,2\end{array}$ & $\begin{array}{r}4,3 \\
-3,8 \\
6,6 \\
6,8 \\
7,5\end{array}$ & $\begin{array}{r}4,7 \\
-1,1 \\
6,4 \\
6,3 \\
7,5\end{array}$ \\
\hline América - Latinoamérica y Caribe & $\begin{array}{l}2,9 \\
2,7 \\
1,4\end{array}$ & $\begin{array}{l}1,3 \\
0,1 \\
2,1\end{array}$ & $\begin{array}{r}0,9 \\
-1,0 \\
3,0\end{array}$ & $\begin{array}{l}2,0 \\
1,0 \\
3,3\end{array}$ \\
\hline 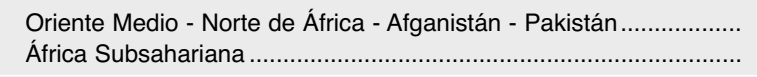 & $\begin{array}{l}2,4 \\
5,2\end{array}$ & $\begin{array}{l}2,6 \\
5,0\end{array}$ & $\begin{array}{l}2,9 \\
4,5\end{array}$ & $\begin{array}{l}3,8 \\
5,1\end{array}$ \\
\hline \multicolumn{5}{|c|}{ IPC (\% variación interanual) } \\
\hline 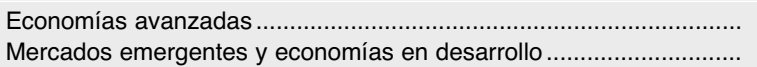 & $\begin{array}{l}1,4 \\
5,9\end{array}$ & $\begin{array}{l}1,4 \\
5,1\end{array}$ & $\begin{array}{l}0,4 \\
5,4\end{array}$ & $\begin{array}{l}1,4 \\
4,8\end{array}$ \\
\hline
\end{tabular}

es uno de los países con mayor dinamismo del PIB en la zona, con crecimientos del PIB estimados en el 2,5 por 100 para 2015 , que ha sido recientemente revisado al alza por el Fondo, al 3,1 por 100. La baja inflación y el riesgo de deflación en los países avanzados siguen motivando recomendaciones de políticas monetarias expansivas por parte del FMI, pero han perdido protagonismo ante el repunte de los precios del petróleo en los últimos meses, tras experimentar caídas muy intensas hasta enero.

La principal novedad es la creciente diferenciación entre las situaciones de los países emergentes. Mientras que China y la India siguen creciendo a tasas cercanas al 7 por 100 (aunque con China en desaceleración) y países como México mantienen un notable crecimiento, varios países en América Latina (Brasil, Argentina, Venezuela) y Rusia están en recesión, muy afectados por las bajadas en los precios de las materias primas y cambios en sus ciclos internos de crédito o «problemas geopolíticos" -en particular las sanciones derivadas del conflicto en Ucrania-. Los países de África Subsahariana, por su parte, mantienen crecimientos apreciables entre el 4,5 y el 5 por 100.

En materia fiscal, los países desarrollados continúan su consolidación fiscal, principalmente impulsada por la mejora del ciclo, con un menor ritmo estructural, mientras que los países emergentes y en desarrollo experimentan un cierto deterioro fiscal, relacionado con los menores niveles de crecimiento y un tono expansivo de su política presupuestaria. El Fondo prevé que la deuda pública en los países desarrollados se estabilice en niveles bastante altos (105 por 100 del PIB de media), mientras que la deuda pública de los países emergentes y en desarrollo mantiene un ligero repunte tras haber bajado muy considerablemente desde el principio de siglo y estabilizarse en niveles inferiores al 40 por 100 en los últimos años.

Un tema que mereció bastante atención fue la evolución cambiaria entre las principales divisas. El euro y el yen han experimentado una considerable depreciación en el último semestre, mientras que el dólar se ha fortalecido. El yuan chino se ha apreciado en paralelo con el dólar. Esta evolución se ha debido principalmente a la divergencia en los $D$ 
signos de la política monetaria, con la zona euro y Japón adoptando políticas no convencionales de compras masivas de activos, mientras que Estados Unidos camina hacia la normalización monetaria, con los mercados previendo la primera subida de tipos de interés de la Reserva Federal en diez años. Los indicadores de inflación siguen en niveles históricamente bajos, con tipos de interés nominales en mínimos históricos y una situación monetaria extraordinaria.

Las principales tensiones y riesgos destacados en las Reuniones de Primavera fueron los riesgos financieros, principalmente la posibilidad de cambios bruscos en los precios de los activos financieros -algunos de ellos en niveles poco normales, afectados por los extraordinariamente bajos tipos de interés-, la incertidumbre derivada de la situación de Grecia, que ha quitado algo de lustre a la incipiente recuperación europea, y riesgos geopolíticos, relacionados con los conflictos de Ucrania y de Siria e Irak.

\section{Líneas de actuación del FMI}

El CMFI (IMFC) tiene como principal objetivo servir de foro de coordinación de las políticas internacionales, pero en la práctica en este ámbito no ejerce el protagonismo único, puesto que el G-20 ha cobrado un rol principal en esta materia, el G-7 sigue ejerciendo una coordinación -aunque más discreta que en el pasado- de las principales potencias industrializadas, y el foro BRICS (Brasil, Rusia, India, China y Sudáfrica) también ha cobrado un creciente papel en los últimos años, para las principales potencias emergentes. La principal función del CMFI ha sido la de marcar la línea de actuación del FMI.

El principal objeto de debate en el CMFI ha sido la reforma del gobierno del Fondo. La reforma de cuotas y gobierno acordada en 2010 todavía no ha sido ratificada por el Congreso de los Estados Unidos. Son más de cuatro años sin que esta ratificación se haya producido, lo cual ha generado una creciente impaciencia en los beneficiarios de la misma. La reforma de 2010 establece tres cambios esenciales:

1. Aumento de los recursos financieros propios: la reforma duplica las cuotas (asimilables al capital de la institución) de los países miembros, que pasan de 238.000 millones de derechos especiales de giro a 477.000 millones (aproximadamente 600.000 millones de euros).

2. Cambios en el peso relativo de los países miembros: se reequilibran las cuotas y el poder de voto, para reflejar mejor las realidades de la economía mundial. Esto beneficia principalmente a los países emergentes, que han crecido más que los países desarrollados en las últimas décadas, pero también a países históricamente infrarrepresentados como España. España pasará, una vez que entre en vigor la reforma de 2010, a una cuota del 2 por 100 del total-actualmente es del 1,69 por 100-.

3. Voz y representación: cambia la estructura del Directorio Ejecutivo, donde se toman las principales decisiones de la institución y se transmite la «VOZ» de los países miembros, para reducir en dos «sillas" ${ }^{2}$ el peso de algunos países europeos sobrerrepresentados de la Europa avanzada. También se eliminaba la distinción entre las «sillas designadas» para los 5 mayores miembros, que no requerían votación. Estas «sillas designadas» pasan ahora a ser elegidas.

La falta de entrada en vigor de la reforma de 2010 supone varios problemas para la institución. El primero es de credibilidad, y afecta tanto al FMI como al G-20, principal impulsor de la reforma y donde se decidieron sus grandes líneas. Una tardanza tan prolongada en aplicar una decisión importante reduce la confianza en que los acuerdos alcanzados tengan valor. El segundo problema es de representatividad o, como afirma el CMFI en su comunicado de abril, de legitimidad. En particular, China, pese a ser la segunda economía $\triangleright$

\footnotetext{
2 Los representantes de los países miembros en el Directorio Ejecutivo del FMI eligen o designan a sus representantes en «sillas» (constituencies en inglés). En el Directorio se sientan 24 Directores Ejecutivos.
} 
mundial por PIB, el primer exportador y primer tenedor de reservas, tiene solamente la sexta mayor cuota, inferior a la del Reino Unido o Francia. Las cuotas en el FMI han sido consideradas políticamente como un símbolo del peso institucional de un país en la economía mundial y en el sistema financiero internacional, y China ha mostrado un creciente interés en un aumento gradual pero significativo de su peso efectivo. Muchos analistas, incluyendo algunos significativos ex altos cargos de Estados Unidos, como Lawrence Summers o Ben Bernanke, han lamentado que este bloqueo institucional puede desviar el poder institucional efectivo hacia instituciones alternativas como el Banco Asiático de Inversión en Infraestructuras, de claro predominio chino, o el Nuevo Banco de Desarrollo (New Development Bank) y el Acuerdo Contingente de Reservas, impulsados por los BRICS, pero con liderazgo efectivo y sede en China.

Otro problema para el Fondo Monetario puede ser de insuficiencia de recursos para un adecuado funcionamiento de la institución. EI Fondo viene utilizando, desde 2009, predominantemente recursos provenientes de los Nuevos Acuerdos de Préstamo (NAP o NAB por sus siglas en inglés), dado el volumen relativamente limitado de las cuotas totales. Los NAB provienen, como las cuotas, del balance de los bancos centrales, pero presentan un inconveniente: deben ser activados cada seis meses mediante una votación del 85 por 100 de los prestamistas, y existe un riesgo de que algunos países, particularmente los que se impacienten por la falta de ratificación de la reforma de 2010, se opongan a su activación. Este riesgo es muy limitado (los BRICS no superan el 15 por 100 de los NAB, y el resto de los prestamistas no suele plantear pegas) pero existe.

Para mitigar estos problemas, el personal del FMI había planteado en enero la posibilidad de unas «medidas transitorias» (interim steps) que permitieran avanzar en los puntos más importantes de la reforma de 2010 salvo en la cuestión de las sillas designadas, que requiere una enmienda al tratado internacional constitutivo del
FMI, y por lo tanto su refrendo parlamentario. De este modo, se podría avanzar sin tener que pasar por todos los Parlamentos de los países miembros.

No obstante, la Administración de los Estados Unidos (el Poder Ejecutivo) mostró su firme compromiso con la ratificación de la reforma de 2010 en el Poder Legislativo, y su confianza en la viabilidad de llegar a un acuerdo, pese a que el Ejecutivo y el Legislativo son en este momento de diferente color político. Las medidas interinas tampoco recibieron apoyo por parte de los países emergentes, que mostraron su preferencia por una ratificación íntegra de la reforma antes que una aplicación parcial transitoria, que podría reducir los incentivos para la ratificación en EEUU. De este modo, el CMFI acordó en la práctica dar más tiempo a los Estados Unidos para conseguir la ratificación legislativa de la reforma. Las medidas interinas siguen siendo una posibilidad, pero de momento no se ha querido recurrir a ellas.

En este contexto, el CMFI no ha decidido cambios significativos en las principales políticas del Fondo -supervisión de políticas económicas y su marco de préstamos- en esta ocasión, pero sí ha marcado la agenda de trabajo. En particular, se avecinan dos cuestiones muy significativas en este año: la composición de la cesta de los derechos especiales de giro, con la aspiración de China a formar parte de la misma, y el debate sobre el marco de préstamos del Fondo, que podría retomar el polémico intento de 2014 del personal del Fondo de condicionar los préstamos de gran tamaño a reestructuraciones de deuda si hay «dudas» sobre su sostenibilidad.

EI CMFI ha prestado particular atención en estas reuniones a los países en desarrollo. Para conceder un tratamiento particularmente favorable a los países afectados por el brote de ébola y a casos similares, el CMFI ha refrendado el establecimiento de un fondo especial para el Alivio y Contención de Catástrofes (Catastrophe Containment and Relief Trust), que permitirá incluso sufragar reducciones de la deuda de estos países con el FMI. El CMFI también ha mostrado su apoyo para la $\triangle$ 
implicación plena del FMI en las tres grandes conferencias vinculadas con el llamado «nuevo multilateralismo para un futuro sostenible»: la Cumbre de Financiación del Desarrollo de julio de 2015 en Addis Abeba (Etiopía), la Cumbre de Nueva York (EEUU) en septiembre de 2015 para establecer unos Objetivos de Desarrollo Sostenible que sustituyan a los llamados Objetivos del Milenio, y la Conferencia de las Partes (COP-21) sobre Cambio Climático en París en diciembre.

\section{4. $\mathrm{G}-20$}

Como parte del calendario muy intenso de reuniones en la semana de las Asambleas, se celebran reuniones de ministros del G-20, en las que España participa como invitado permanente, estatus asimilable al de miembro en la práctica, con voz y capacidad de negociación en los comunicados, y presencia en todos los grupos de trabajo técnicos. Otros grupos, como el G-7, los BRICS o el G-24 (que reúne a los principales países en desarrollo), también celebran reuniones de alto nivel en la semana de Asambleas, dentro de una geometría variable de coordinación internacional cuya complejidad y carga de trabajo ha ido aumentando en los últimos años.

El comunicado del G-20 destaca por su tono más optimista que en ocasiones anteriores, reconociendo que hay menores riesgos que en el pasado reciente (risks are more balanced), pese a la desaceleración en los países emergentes. Destaca que la bajada de los precios del petróleo es positiva para la economía mundial, pero el grupo advierte de un elevado número de riesgos que le preocupan: la volatilidad en los tipos de cambio y en los flujos de capitales internacionales, relacionada con la creciente divergencia en los signos de las políticas monetarias de las principales áreas económicas, la creciente volatilidad en los mercados financieros, el riesgo de una inflación muy baja por tiempo prolongado, desequilibrios globales o en patrones domésticos de crecimiento, elevada deuda pública y tensiones geopolíticas.
En 2014, bajo presidencia del G-20 de Australia, el principal tema fue el fomento del crecimiento a medio y largo plazo mediante reformas estructurales de los países miembros, que se plasmarían en unos documentos de Estrategias de Crecimiento. Este año es el año de puesta en práctica de los planes expuestos en las mencionadas Estrategias (the year of Implementation, como ha considerado la presidencia turca del G-20 en 2015), así como de su actualización. Para ello, los países están efectuando revisiones mutuas (peer reviews) de sus estrategias y señalando posibles ámbitos de mejora, con la ayuda de las organizaciones internacionales.

Otro tema destacado en este año es el fomento de la inversión. Los países miembros van a presentar estrategias para mejorar los climas de inversión, fomentar la eficiencia de la inversión pública en infraestructuras y apoyar la financiación de la inversión a largo plazo de las pequeñas y medianas empresas. El G-20 también quiere promover buenas prácticas en el uso de los partenariados público-privados para inversión pública, y maximizar el uso de los recursos de los bancos multilaterales de desarrollo. También se prevé que entre en funcionamiento a lo largo de este año un centro de fomento de las infraestructuras con sede en Australia, el Global Infrastructure Hub.

Una de las funciones principales del G-20 es la de impulsar la agenda global de regulación financiera, en estrecha colaboración con el Consejo de Estabilidad Financiera (Financial Stability Board, FSB, por sus siglas en inglés). Se aspira a llegar para la cumbre del G-20 de Antalya (octubre de 2015) a un acuerdo definitivo sobre un estándar internacional de capitalización adicional en los bancos sistémicos globales, denominado "capacidad de absorción de pérdidas» (Total Loss Absorbing Capacity, o TLAC). Este es quizá el punto más sensible y con mayor impacto sobre el sistema financiero, y por ello se está prestando particular atención a estudios de impacto para calibrar el acuerdo final. También se continuará con los trabajos en la regulación de las cámaras de compensación, $D$ 
requerimientos de capitalización para aseguradoras sistémicas, banca en la sombra (shadow banking) y derivados negociados fuera de los mercados oficiales (Over-the-Counter, OTC).

En estos últimos años, también se han efectuado importantes progresos en materia de tributación fiscal internacional, con el impulso conjunto del G-20 y de la OCDE. Se espera llegar antes de la cumbre a un acuerdo definitivo sobre el Plan de Acción sobre erosión de bases fiscales impositivas y desviación de beneficios (Base Erosion and Profit Shifting, BEPS), que permitirá reducir las posibilidades de arbitraje fiscal perjudicial. El año pasado se llegaron a acuerdos extremadamente importantes sobre intercambio automático de información fiscal, que están en proceso de puesta en práctica, que será largo, dada la importante complejidad técnica.

\section{Conclusión}

Las Asambleas de Primavera se han visto marcadas por la creciente frustración por el bloqueo en la adopción de las reformas de cuotas y gobierno del Fondo Monetario Internacional. Se ha decidido conceder más tiempo para la ratificación por parte de todos los miembros (en concreto de Estados Unidos). La falta de ratificación de las reformas supone un cierto desgaste para la institución, y conlleva algunos riesgos para los mismos recursos del Fondo. No obstante, el Fondo Monetario sigue disponiendo de la posibilidad de adoptar medidas interinas, y en caso de que surgiera una clara necesidad en caso de crisis, parece claro que se podrían adoptar esas alternativas con cierta rapidez.

A lo largo del año, el reconocimiento del creciente peso de China va a planear sobre varias reuniones internacionales, no sólo por la reforma de cuotas y gobierno del FMI, sino también por la cuestión de la composición de la cesta de los derechos especiales de giro. China ha mostrado su interés político en que su divisa forme parte de la cesta, y supondría un cambio muy significativo en el sistema monetario internacional.

Los crecientes problemas en varios países emergentes todavía no han motivado solicitudes de programas grandes con el Fondo. Los niveles de reservas son todavía elevados, y no se ha producido un fenómeno de salidas de capitales a gran escala. $\mathrm{Si}$ se confirma la senda de normalización monetaria de los Estados Unidos, y se producen subidas significativas de tipos de interés, se pondrá a prueba la adecuación del marco de préstamos del FMI a un nuevo ciclo de flujos de capitales.

\section{Bibliografía}

[1] COMITÉ MONETARIO Y FINANCIERO INTERNACIONAL (2015). Comunicado de la $31^{a}$ reunión del CMFI. 18 de abril de 2015. Disponible en: http://www.imf.org/external/np/cm/2015/041815.htm

[2] FONDO MONETARIO INTERNACIONAL (2015). Perspectivas de la Economía Mundial. Abril de 2015. Disponible en: http://www.imf.org/external/spanish/pubs/ft/weo/ 2015/ 01/pdf/texts.pdf

[3] FONDO MONETARIO INTERNACIONAL (2015). IMF Executive Board Reports to the Board of Governors on the 2010 Reforms and the Fifteenth General Review of Quotas. Nota de Prensa (Press Release $n^{\circ}$. 15/20). 28 de enero de 2015. Disponible en:

http://www.imf.org/external/np/sec/pr/2015/ pr1520.htm

[4] G-20 (2015). Comunicado de los Ministros de Finanzas y Gobernadores de Bancos Centrales. 16-17 de abril de 2015. Disponible en: https://g20.org/wp-content/uploads/2015/04/ April-G20-FMCBG-Communique-Final.pdf

[5] SUMMERS, L. (2015). «Time US leadership woke up to new economic era». Editorial en el Financial Times de Londres. 5 de abril de 2015. 